\title{
Proton and Neutron Electromagnetic Form Factors Based on Bound System in $3+1$ Dimensional QCD
}

\author{
Teruo Kurai \\ 1-32-1 Nishifu-machi, Fuchu-shi, Tokyo, Japan \\ Email: kurai.teruo@topaz.plala.or.jp
}

How to cite this paper: Kurai, T. (2020) Proton and Neutron Electromagnetic Form Factors Based on Bound System in 3 +1 Dimensional QCD. Journal of Modern Physics, 11, 741-765. https://doi.org/10.4236/jmp.2020.115048

Received: March 27, 2020

Accepted: May 18, 2020

Published: May 21, 2020

Copyright (๑) 2020 by author(s) and Scientific Research Publishing Inc. This work is licensed under the Creative Commons Attribution International License (CC BY 4.0).

http://creativecommons.org/licenses/by/4.0/ (c) (i) Open Access

\begin{abstract}
We propose a new description of a nucleon as a pair of pions. The baryon number of our description of nucleon is not 1 but 0 . However, this is probable because the proton spin crisis shows that the baryon spin cannot tell the number of composing quarks anymore. Because we use the derived pion wave function to describe a nucleon, our description has automatically the pionic degree of freedom and can be compared to the constituent quark model. Using this description, we investigate the electric charge and magnetization density functions of protons and neutrons. The electric charge density function of neutron is quite similar to those of Galster model and Maints data except the magnitude of singularity. The density functions of proton also show the similar behavior as those of Kelly's except near origin. Taking the Fourier transform of the density functions, we obtain the Sachs electromagnetic form factors that can be compared to those in the parametrization derived by Ye et al.
\end{abstract}

\section{Keywords}

Density Function, Electromagnetic Form Factor

\section{Introduction}

The proton and neutron electromagnetic form factors (e.m. FFs) are key components for understanding the charge and magnetization distributions within nucleons. In the past 20 years, a new generation of experiments, frequently utilizing polarization of freedom, has provided new knowledge regarding our understanding of the form factors [1] [2]. The parametrization work proposed by Ye et al. [3] to analyze the form factors including uncertainties used the com- 
plete world data set for electron scattering and applied their best knowledge of two photon exchange (TPE) corrections. From the viewpoint of the charge and magnetization distributions within nucleons, which are principal reasons to investigate e.m. FFs, we need to investigate the wave functions (WFs) of nucleon directly. Under the constituent quark model (CQM), Chung and Coester [4] developed the light-front calculation of the nucleon FF using a Gaussian WF in the quark internal (transverse) momentum variables. This model yields good agreement with the observed $G_{E}^{[P]} / G_{M}^{[P]}$ ratio, but its nucleon FF decreases too quickly at larger $|\vec{Q}|^{2}$. Schlumpf [5] used the power law dependence of the quadratic internal momentum variables in the nucleon light-front WF and showed reasonable results for the power behavior of the FF at larger $|\vec{Q}|^{2}$. The WF of Schlumpf was used by Frank [6] and Millar [7]. Cardarell [8] used the rest-frame WF obtained by the Capstich-Isgur model [9] and found it to yield a significant content of high-momentum components, which are generated by the short range portion of quark-quark interactions. A comparable amount of high-momentum components in the nucleon WF was obtained in the Goldstone-boson-exchange quark model (GBE CQM), and this led to the nucleon e.m. FFs in point-like form [10] [11] [12] [13] [14]. The covariant CQM calculation yielding fairly good agreement with the nucleon FF was performed by Gross and Agbite [15]. For the pion cloud model, Miller [16] performed a light-front cloudy bag model calculation. This chiral quark model includes the perturbative pions, and is improved by Faessler [17]. A non-perturbative approach that combines both quark and pion degrees of freedom and interpolates between CQM and the SKyrum model (where the nucleon appears as a soliton solution of an effective nonlinear pion field) is the chiral soliton model ( $\chi \mathrm{QSM})$, in which the baryon density is not exactly Gaussian but is quite close to it. Another approach to the estimation of Sachs e.m. FFs results from the generalized parton distribution scheme (GPD) [18] [19] [20]. In this scheme, the three-parameter modified Regge mode provides a good description in the range of low $|\vec{Q}|^{2}$ to large $|\vec{Q}|^{2}$, but the ratio of $G_{E}^{[P]} / G_{M}^{[P]}$ becomes negative beyond $8\left(\mathrm{GeV}^{2}\right)$, which does not fit the data [21]. Because this analysis was conducted in momentum space, it was necessary to take the Fourier Transform to obtain the charge and magnetization density functions (distribution functions). In this paper, we propose a new description of a nucleon, i.e., a pion pair, in configuration space (r-space) and show that the charge and magnetization density functions follow directly from this description. Taking the Fourier transform, we derive the Sachs e.m. FFs, which are comparable to those in Ye et al. [3]. This means that we can investigate the Sachs e.m. FFs and the charge and magnetization density functions with opposite ordering, in contrast to Kelly's way [22].

To clarify, we list here the symbols and parameters.

$\rho_{c h}^{[P]}, \rho_{m}^{[P]}$ : electric charge and magnetization density functions of proton.

$\rho_{c h}^{[N]}, \rho_{m}^{[N]}$ : electric charge and magnetization density functions of neutron. 
$\tilde{\rho}_{c h}^{[P]}, \tilde{\rho}_{m}^{[P]}$ : proton intrinsic FFs that are Fourier transform of electric charge and magnetization density functions.

$\tilde{\rho}_{c h}^{[N]}, \tilde{\rho}_{m}^{[N]}$ : neutron intrinsic FFs that are Fourier transform of electric charge and magnetization density functions.

$n_{p}, n_{N}$ : parameters of the power of exponentials of proton and neutron these are used for both density functions and Sachs e.m. FFs.

$m_{P}, m_{N}$ : parameters of the coefficient of $r$ or $|\vec{Q}|^{2}$.

$\beta:$ range parameter for density functions.

$p$ : parameter of the coefficient of logarithmic term of Sachs e.m. FFs.

\section{Derivation}

\subsection{Basic Concept}

To date, there have been several proposed descriptions for a nucleon. One of these is the pion cloud model, which introduces an elementary, perturbative pion couple to the constituent quark model (CQM) such that chiral symmetry is restored. Noting the fact that the contribution of quark spins to the spin of a proton is small, i.e., the proton spin crisis [23], it is a fair consideration that nucleons are described only by the pion pair as far as the e.m. FFs are concerned. Baryon number of our case is not 1 but 0 , however, proton crisis tells that the composing quark intrinsic spin does not contribute to that of baryon. This indicates that the baryon spin cannot tell the number of the composing quarks. The reason is following. It is quite reasonable consideration that the spin of all baryons are not determined by their composing quark intrinsic spin because all baryons decay to proton as a final state. The baryon number 1 comes from the assumption that the number of quarks is three for all baryons, and this assumption is based on the consideration that the composing quark intrinsic spin determines that of baryon. Proton spin crisis shows that this assumption lost the experimental support. Then, it is probable to consider that baryon number is not 1. Thus, we propose the description of nucleon as a pion pair even though baryon number is 0 . Normally, the simplest description of a proton is uud (up, up, down) and that of a neutron is udd (up, down, down), while our description of proton is a $\pi^{+}-\pi^{0}$ pair composed of $u \bar{d}-(u \bar{u}+d \bar{d}) / \sqrt{2}$, and that of a neutron is a $\pi^{+}-\pi^{-}$pair composed of $u \bar{d}-\bar{u} d$. The most important feature of the WFs is to describe the e.m. FFs because the electric charge and the magnetization density functions (distribution functions) are directly connected to them, as shown by Kelly [22]. We derived the pion $\left(\pi^{+}\left(\pi^{-}\right)\right)$WF based on the hadronic operator proposed by Suura [24] [25] in a previous paper [26]. The derived charged pion WF that we use in this paper is Gaussian/ $r$ in configuration space ( $r$ space) thus the density function is Gaussian $\times F\left(1, \frac{3}{2} ; Q^{2}\right)$ in momentum space as shown later section. $F\left(1, \frac{3}{2} ; Q^{2}\right)$ is the one of Kummer's conflu- 
ent hyper geometric series. Therefore this is quite similar to the constituent quark model (CQM) using with Schlumpf's $Q^{2}$ polynomials momenta. Therefore we can expect our e.m. FFs become similar to these of CQM except their behavior in larger $Q^{2}$ region because two pion moves independently in this region as we explain later section. Thus, as far as e.m. FFs are concerned, we consider a proton and a neutron to be described as $\pi^{+}-\pi^{0}$ and $\pi^{+}-\pi^{-}$, respectively.

\subsection{Basic Concept of Evaluation}

The Bethe-Salpeter-like amplitude of the hadronic operator applied in this paper is defined as

$$
\chi(1,2)=\langle 0|q(1,2)| P\rangle
$$

where $|0\rangle$ and $|P\rangle$ denote a vacuum and the physical state, respectively.

The gauge-invariant bi-local operator $q(1,2)$ is defined in the non-Abelian gauge field as

$$
q(1,2)=T_{r}^{c} q_{\beta}^{+}(2) P \exp \left(i g \int_{1}^{2} \mathrm{~d} \vec{x} \vec{A}^{a}(x) \frac{\lambda_{a}}{2}\right) q_{\alpha}(1)
$$

here $\alpha$ and $\beta$ denote the Dirac indices, $P$ denotes the path ordering, and the $\frac{\lambda_{a}}{2}$ components are generators of the adjoint representation of the SU $(\mathrm{N})$. color gauge group. The trace is calculated for color spin a. Suura first proposed this definition [24] [25], and later applied it to the case of the light meson mass spectra, the t'Hooft model, and the pion e.m. FF [26] [27] [28]. In the case of pions, quarks (and antiquarks) are specified as $u(\bar{u})$ and $d(\bar{d})$, however, as we previously described [26], we obtained the pion wave function with eigenvalues and eigen functions of the equation of motion for a $\chi(1,2)$ system. Thus, we can describe the $\pi^{+}-\pi^{0}$ pair as $\chi(1,2)$ and $\chi(3,4)$. Here, we describe $\pi^{0}$ as just one Bethe-Salpeter-like amplitude even though $\pi^{0}$ is desdribed as $(u \bar{u}+d \bar{d}) / \sqrt{2}$. As mentioned in section 2, the derived pion WF in Ref. [26] is for a charged pion. We cannot expect the WF of $\pi^{0}$ to be the same as that of charged pion, but the mass of $\pi^{0}$ is very close to that of charged pion. Thus, we assume that $\pi^{0}$ is also described as the same form of the Bethe-Salpeter-like amplitude of the hadronic operator as that of the charged pion.

\subsection{Evaluation}

The basic concept of our evaluation is as follows.

Because baryons are represented as initially binding meson pairs as described in subsection 5, at $|\vec{Q}|^{2}=0$ and for the small $|\vec{Q}|^{2}$ case, the $\pi^{+}-\pi^{0}$ pair has the same origin. For simplicity, both of quarks are in the same position. However, when $|\vec{Q}|^{2}$ is large, both $\pi^{+}$and $\pi^{0}$ gradually move freely with respect to each other and both $\pi^{+}$and $\pi^{0}$ move totally independent of each other, which is as the same concept as asymptotic freedom. 


\subsubsection{Density Functions}

$$
\begin{aligned}
q(1 ; 2,3)= & \frac{1}{2}\left[\left(T_{r}^{c} q^{\dagger}(2) P \exp \left(i g \int_{1}^{2} \mathrm{~d} \vec{x} \vec{A}^{a}(x) \frac{\lambda_{a}}{2}\right) q(1)\right)^{\left(\pi^{+}\right)}\right. \\
& \left.+\left(T_{r}^{c} q^{\dagger}(3) P \exp \left(i g \int_{1}^{3} \mathrm{~d} \vec{x} \vec{A}^{a}(x) \frac{\lambda_{a}}{2}\right) q(1)\right)^{\left(\pi^{0}\right)}\right] \\
& +\frac{1}{2}\left[\left(T_{r}^{c} q^{\dagger}(2) P \exp \left(i g \int_{1}^{2} \mathrm{~d} \vec{x} \vec{A}^{a}(x) \frac{\lambda_{a}}{2}\right) q(1)\right)^{\left(\pi^{0}\right)}\right. \\
& \left.+\left(T_{r}^{c} q^{\dagger}(3) P \exp \left(i g \int_{1}^{3} \mathrm{~d} \vec{x} \vec{A}^{a}(x) \frac{\lambda_{a}}{2}\right) q(1)\right)^{\left(\pi^{+}\right)}\right]
\end{aligned}
$$

At first $\left(|\vec{Q}|^{2}=0\right)$, the $\pi^{+}-\pi^{0}$ pair is described as where $\left(\pi^{+}\right),\left(\pi^{0}\right)$ means that each described hadronic operator corresponds to $\pi^{+}$and $\pi^{0}$, respectively.

Note that Equation (3) promises the evenly charged wave function and that the total factor $\frac{1}{2}$ keeps proton charge at $+e$, and that the positions of both $\pi^{+}$ and $\pi^{0}$ quarks are at the same point $\left(|\vec{Q}|^{2}=0\right)$.

The latter $\frac{1}{2}$ part is essentially same as the former $\frac{1}{2}$ part when we do not consider the charge distribution. Because our concern is the wave function of the $\pi^{+}-\pi^{0}$ system, the Bethe-Salpeter-like amplitude can be defined as

$$
\chi(1 ; 2.3)=\langle 0|q(1 ; 2.3)| P\rangle
$$

where

$$
q(1 ; 2,3)=q(1,2)+q(1,3)
$$

here, we dropped the factor $\frac{1}{2}$ for simplicity. When considering charge distribution function, we consider this factor again.

Then equation of motion of $q(1 ; 2,3)$ becomes

$$
\begin{aligned}
i \frac{\partial}{\partial t} q(1 ; 2,3)= & i \frac{\partial}{\partial t} q(1,2)+i \frac{\partial}{\partial t} q(1,3) \\
= & -i \vec{\alpha} \cdot \vec{\nabla}(2) q(1,2)-q(1,2) i \vec{\alpha} \cdot \vec{\nabla}(1)+g \int_{1}^{2} \mathrm{~d} \vec{x} q_{\vec{E}}(1,2 ; x) \\
& +g \vec{\alpha} \cdot \int_{1}^{2} \mathrm{~d} \vec{x} \times q_{\vec{B}}(1,2 ; x)-i \vec{\alpha} \cdot \vec{\nabla}(3) q(1,3)-q(1,3) i \vec{\alpha} \cdot \vec{\nabla}(1) \\
& +g \int_{1}^{3} \mathrm{~d} \vec{x} q_{\vec{E}}(1,3 ; x)+g \vec{\alpha} \cdot \int_{1}^{3} \mathrm{~d} \vec{x} \times q_{\vec{B}}(1,3 ; x) \\
& q_{\vec{o}}(1, s ; x)=q^{+}(s) U(s, x) \vec{O}^{a} \frac{\lambda_{a}}{2} U(x, 1)
\end{aligned}
$$

where

$$
U(s, 1) \equiv P \exp \left(i g \int_{1}^{s} \mathrm{~d} \vec{x} \vec{A}^{a}(x) \frac{\lambda_{a}}{2}\right)
$$




$$
\alpha^{k}=\gamma^{0} \gamma^{k}
$$

$O$ is any operator, $s=2,3$ and $\gamma^{\mu}$ is $\gamma$ is matrices.

We previously obtained the equation of $i \frac{\partial}{\partial t} q(1, s)$ [28].

Because we consider every quark mass to be zero, the center of mass coordinate and two relative coordinates can be written

$$
\begin{aligned}
\vec{G} & =\frac{\vec{r}_{1}+\vec{r}_{2}+\vec{r}_{3}}{3} \\
\vec{r}^{(1)} & =\vec{r}_{2}-\vec{r}_{1}, \vec{r}^{(2)}=\vec{r}_{3}-\vec{r}_{1}
\end{aligned}
$$

Then

$$
\begin{aligned}
& \vec{r}_{1}=\vec{G}-\frac{1}{3} \vec{r}^{(1)}-\frac{1}{3} \vec{r}^{(2)} \\
& \vec{r}_{2}=\vec{G}+\frac{2}{3} \vec{r}^{(1)}-\frac{1}{3} \vec{r}^{(2)} \\
& \vec{r}_{3}=\vec{G}-\frac{1}{3} \vec{r}^{(1)}+\frac{2}{3} \vec{r}^{(2)}
\end{aligned}
$$

Thus, the derivatives are

$$
\begin{aligned}
\vec{\nabla}(1) & =\frac{\partial}{\partial \vec{r}}=\frac{1}{3} \frac{\partial}{\partial \vec{G}}-\frac{\partial}{\partial \vec{r}^{(1)}}-\frac{\partial}{\partial \vec{r}^{(2)}} \\
\vec{\nabla}(2) & =\frac{1}{3} \frac{\partial}{\partial \vec{G}}+\frac{\partial}{\partial \vec{r}^{(1)}} \\
\vec{\nabla}(3) & =\frac{1}{3} \frac{\partial}{\partial \vec{G}}+\frac{\partial}{\partial \vec{r}^{(2)}}
\end{aligned}
$$

Remembering that $\vec{\alpha} \cdot \vec{\nabla}(s)=\alpha^{l} \frac{\partial}{\partial r_{s}^{l}} \quad(s=1,2,3)$, the kinetic term becomes

$$
\begin{aligned}
& -i \vec{\alpha} \cdot \vec{\nabla}(2) q(1,2)-q(1,2) i \vec{\alpha} \cdot \vec{\nabla}(1) \\
& =-\frac{i}{3}\left\{\alpha^{l} \frac{\partial}{\partial G^{l}}, q(1,2)\right\}_{+}-i\left[\alpha^{l} \frac{\partial}{\partial r^{(1) l}}, q(1,2)\right]+q(1,2) i \alpha^{l} \frac{\partial}{\partial r^{(2) l}}
\end{aligned}
$$

Similarly

$$
\begin{aligned}
& -i \vec{\alpha} \cdot \vec{\nabla}(3) q(1,3)-q(1,3) i \vec{\alpha} \cdot \vec{\nabla}(1) \\
& =-\frac{i}{3}\left\{\alpha^{l} \frac{\partial}{\partial G^{l}}, q(1,3)\right\}_{+}-i\left[\alpha^{l} \frac{\partial}{\partial r^{(2) l}}, q(1,3)\right]+q(1,3) i \alpha^{l} \frac{\partial}{\partial r^{(1) l}}
\end{aligned}
$$

We consider the gauge field string only for the straight line case. Thus, the hadronic operator $q(1,2)$ is decomposed in the relative coordinate system as

$$
q(1,2)=q_{0}\left(r^{(1)}\right)+i \vec{\alpha} \cdot \hat{r}^{(1)} q_{1}\left(r^{(1)}\right)+\beta q_{2}\left(r^{(1)}\right)+\beta\left(i \vec{\alpha} \cdot \hat{r}^{(1)}\right) q_{3}\left(r^{(1)}\right)
$$

where $r^{(1)}=\left|\vec{r}^{(1)}\right|$

Because $q_{0}\left(r^{(1)}\right)=0, q_{1}\left(r^{(1)}\right)=0$ (as we previously showed [26]), only term we have to deal with is $i \frac{\partial}{\partial r^{(2) l}}\left(\beta\left(i \vec{\alpha} \cdot \hat{r}^{(1)}\right) q_{3}\left(r^{(1)}\right)\right)$. 


$$
\begin{gathered}
-\beta i \alpha^{l} \frac{\partial}{\partial r^{(2) !}} i \alpha^{m} \frac{x^{(1) m}}{r^{(1)}}=\beta \alpha^{l} \alpha^{m} \frac{\partial}{\partial x_{l}^{(2)}}\left(\frac{x_{m}^{(1)}}{r^{(1)}}\right)=0 \\
\frac{\partial}{\partial x^{(2) !}} q_{s}\left(r^{(1)}\right)=0 \quad(s=2,3)
\end{gathered}
$$

here, we denote that $r^{(p) l}=x_{l}^{(p)} \quad(p=1,2)$, so that $r^{(1)}=\sqrt{x_{1}^{(1) 2}+x_{2}^{(1) 2}+x_{3}^{(1) 2}}$

Thus,

$$
q(1,2) i \alpha^{l} \frac{\partial}{\partial r^{(2) l}}=0
$$

Similarly

$$
q(1,3) i \alpha^{l} \frac{\partial}{\partial r^{(1) l}}=0
$$

Therefore, the kinetic terms in the relative coordinate system become

$$
\begin{aligned}
& -i \vec{\alpha} \cdot \vec{\nabla}(2) q(1,2)-q(1,2) i \vec{\alpha} \cdot \vec{\nabla}(1)-i \vec{\alpha} \cdot \vec{\nabla}(3) q(1,3)-q(1,3) i \vec{\alpha} \cdot \vec{\nabla}(1) \\
& =-i\left[\alpha^{l} \frac{\partial}{\partial r^{(1) l}}, q(1,2)\right]-i\left[\alpha^{l} \frac{\partial}{\partial r^{(2) l}}\right]
\end{aligned}
$$

The integral terms in relative coordinate system become

$$
\begin{gathered}
g \int_{1}^{2} \mathrm{~d} \vec{x} q_{\vec{E}}(1,2 ; x)=-\frac{g^{2}}{2} \int_{0}^{r^{(1)}} \mathrm{d} z q\left(r^{(1)}-z\right)\left(r^{(1)}-z\right) q(z) \\
g \vec{\alpha} \cdot \int_{1}^{2} \mathrm{~d} \vec{x} \times q_{\vec{B}}(1,2 ; x)=\frac{g^{2}}{2} \int_{-\infty}^{t} \mathrm{~d} t^{\prime}\left(\vec{\alpha} \cdot \hat{r}^{(1)}\right) \delta\left(t-t^{\prime}\right) \int_{0}^{r^{(1)}} \mathrm{d} z q\left(t^{\prime}, r^{(1)}-z\right) q\left(t^{\prime}, z\right) \\
g \int_{1}^{3} \mathrm{~d} \vec{x} q_{\vec{E}}(1,3 ; x)=-\frac{g^{2}}{2} \int_{0}^{r^{(2)}} \mathrm{d} z q\left(r^{(2)}-z\right)\left(r^{(2)}-z\right) q(z) \\
g \vec{\alpha} \cdot \int_{1}^{3} \mathrm{~d} \vec{x} \times q_{\vec{B}}(1,3 ; x)=\frac{g^{2}}{2} \int_{-\infty}^{t} \mathrm{~d} t^{\prime}\left(\vec{\alpha} \cdot \hat{r}^{(2)}\right) \delta\left(t-t^{\prime}\right) \int_{0}^{r^{(2)}} \mathrm{d} z q\left(t^{\prime}, r^{(2)}-z\right) q\left(t^{\prime}, z\right)
\end{gathered}
$$

We obtained these equations were obtained previously [26].

Thus, the equation of motion for $q(1 ; 2,3)$ is expressed by the following independent equations in the relative coordinate system.

$$
\begin{aligned}
i \frac{\partial}{\partial t} q\left(r^{(1)}\right)= & -i\left[\vec{\alpha} \cdot \vec{\nabla}_{r^{(1)}}, q\left(r^{(1)}\right)\right]-\frac{g^{2}}{2} \int_{0}^{r^{(1)}} \mathrm{d} z q\left(r^{(1)}-z\right)\left(r^{(1)}-z\right) q(z) \\
& +\frac{g^{2}}{2} \int_{-\infty}^{t} \mathrm{~d} t^{\prime}\left(\vec{\alpha} \cdot \hat{r}^{(1)}\right) \delta\left(t-t^{\prime}\right) \int_{0}^{r^{(1)}} \mathrm{d} z q\left(t^{\prime}, r^{(1)}-z\right) q\left(t^{\prime}, z\right) \\
i \frac{\partial}{\partial t} q\left(r^{(2)}\right)= & -i\left[\vec{\alpha} \cdot \vec{\nabla}_{r^{(2)}}, q\left(r^{(2)}\right)\right]-\frac{g^{2}}{2} \int_{0}^{r^{(2)}} \mathrm{d} z q\left(r^{(2)}-z\right)\left(r^{(2)}-z\right) q(z) \\
& +\frac{g^{2}}{2} \int_{-\infty}^{t} \mathrm{~d} t^{\prime}\left(\vec{\alpha} \cdot \hat{r}^{(2)}\right) \delta\left(t-t^{\prime}\right) \int_{0}^{r^{(2)}} \mathrm{d} z q\left(t^{\prime}, r^{(2)}-z\right) q\left(t^{\prime}, z\right)
\end{aligned}
$$

Thus, the WF of the $\pi^{+}-\pi^{0}\left(\pi^{-}\right)$pair, $\chi_{\text {pair }}$, is described as

$$
\chi_{\text {pair }}=c_{1} \chi_{\pi}\left(r^{(1)}\right)+c_{2} \chi_{\pi}\left(r^{(2)}\right)
$$

where 


$$
\chi_{\pi}(r)=\text { const } \frac{e^{-\frac{g^{2} L_{1}}{8} r^{2}}}{r}
$$

We obtained the pion WF (Equation (24)) in our prior analysis [26].

Therefore, the magnetization density function and the basis of the electric charge density function of a proton are described as

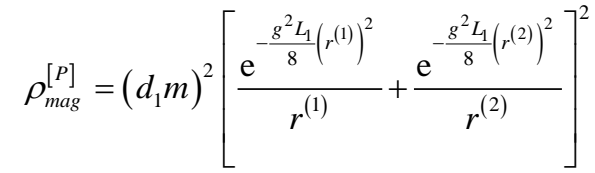

$$
\begin{aligned}
& \text { Basis } \rho_{c h}^{[P]}=\left(d_{2} e\right)^{2}\left[\frac{\mathrm{e}^{-\frac{g^{2} L_{1}}{8}\left(r^{(1)}\right)^{2}}}{r^{(1)}}+\frac{\mathrm{e}^{-\frac{g^{2} L_{1}}{8}\left(r^{(2)}\right)^{2}}}{r^{(2)}}\right]^{2}
\end{aligned}
$$

here, we combine the latter part of Equation (3) so that the correct magnetization and charge of proton are obtained in Equation (25) and Equation (26). This is because we dropped the factor $\frac{1}{2}$ in the derivation.

By definition, $\vec{r}^{(1)}$ and $\vec{r}^{(2)}$ have the same origin. Thus, considering the direction of momentum $\vec{Q}$ to be the z-axis, $\vec{r}^{(1)}$ and $\vec{r}^{(2)}$ can be expressed by polar coordinates as

$$
\vec{r}^{(1)}=\left(r^{(1)}, \theta_{1}, \phi_{1}\right), \quad \vec{r}^{(2)}=\left(r^{(2)}, \theta_{2}, \phi_{2}\right)
$$

Denoting the angle between $\vec{r}^{(1)}$ and $\vec{r}^{(2)}$ as $\theta$ and $\vec{r}^{(1)}$ to $\vec{r}$, that is, considering $\vec{r}^{(1)}$ as $\vec{r}$, the magnetization density function of a proton is written

$$
\rho_{m}^{[P]}=\left(d_{1} m\right)^{2} \int_{0}^{\pi} \sin \theta_{1} \mathrm{~d} \theta_{1} \int_{0}^{2 \pi} \mathrm{d} \phi_{1}\left[\frac{\mathrm{e}^{-\frac{g^{2} L_{1}}{8} r^{2}}}{r}+\frac{\mathrm{e}^{-\frac{g^{2} L_{1}}{8}(r \cos \theta)^{2}}}{r \cos \theta}\right]^{2}
$$

where $\cos \theta=\cos \theta_{2} \cos \theta_{1}+\sin \theta_{2} \sin \theta_{1} \cos \left(\phi_{2}-\phi_{1}\right)$

Taking integration to eliminate the $\theta_{2}$ and $\phi_{2}$ dependence, the actual form of $\rho_{\text {mag }}$ can be written as

$$
\begin{aligned}
& \rho_{m}^{[P]}= \\
& \left(d_{1} m\right)^{2}\left[4 \pi \frac{\mathrm{e}^{-\frac{g^{2} L_{1}}{4} r^{2}}}{r^{2}}+\int_{0}^{\pi} \mathrm{d} \theta_{2} \int_{0}^{2 \pi} \mathrm{d} \phi_{2} \int_{0}^{\pi} \sin \theta_{1} \int_{0}^{2 \pi} \mathrm{d} \phi_{1}\left(2 \frac{\mathrm{e}^{-\frac{g^{2} L_{1}}{8} r^{2}\left(1+(\cos \theta)^{2}\right)}}{r^{2}}+\frac{\mathrm{e}^{-\frac{g^{2} L_{1}}{4}(r \cos \theta)^{2}}}{r^{2}}\right)\right]
\end{aligned}
$$

To obtain the electric charge density function of a proton $\rho_{c h}^{[p]}$, we need more careful consideration because of the asymptotic freedom as mentioned in sec 2-3. As $|\vec{Q}|^{2}$ becomes larger, $r$ is smaller, $\rho_{c h}^{[P]}$ becomes the summation of two independent $\left|\chi_{\pi}(r)\right|^{2}$ terms.

To be precise, $\rho_{c h}^{[P]}$ behaves as

1) as $r \rightarrow 0$, 


$$
\begin{gathered}
\rho_{c h}^{[P]} \rightarrow\left(d_{2} e\right)^{2} 2(4 \pi) \frac{\mathrm{e}^{-\frac{g^{2} L_{1} r^{2}}{4}}}{r^{2}} \\
2) \text { as } r \rightarrow \infty, \\
\rho_{c h}^{[P]} \rightarrow\left(d_{1} m\right)^{2}\left[4 \pi \frac{\mathrm{e}^{-\frac{g^{2} L_{1}}{4} r^{2}}}{r^{2}}+\int_{0}^{\pi} \mathrm{d} \theta_{2} \int_{0}^{2 \pi} \mathrm{d} \phi_{2} \int_{0}^{\pi} \sin \theta_{1} \int_{0}^{2 \pi} \mathrm{d} \phi_{1}\left(2 \frac{\left.\left.\mathrm{e}^{-\frac{g^{2} L_{1} r^{2}\left(1+(\cos \theta)^{2}\right)}{r^{2}}}+\frac{\mathrm{e}^{-\frac{g^{2} L_{1}}{4}(r \cos \theta)^{2}}}{r^{2}}\right)\right]}{}\right.\right.
\end{gathered}
$$

The angular integrations become

$$
\begin{gathered}
\int_{0}^{\pi} \mathrm{d} \theta_{2} \int_{0}^{2 \pi} \mathrm{d} \phi_{2} \int_{0}^{\pi} \sin \theta_{1} \int_{0}^{2 \pi} \mathrm{d} \phi_{1} \frac{\mathrm{e}^{-\frac{g^{2} L_{1}}{8} r^{2}\left(1+(\cos \theta)^{2}\right)}}{r^{2} \cos \theta}=4 \pi^{3} \frac{\mathrm{e}^{-\frac{g^{2} L_{1}}{8} r^{2}}}{r^{2}} \\
\int_{0}^{\pi} \mathrm{d} \theta_{2} \int_{0}^{2 \pi} \mathrm{d} \phi_{2} \int_{0}^{\pi} \sin \theta_{1} \int_{0}^{2 \pi} \mathrm{d} \phi_{1} \frac{\mathrm{e}^{-\frac{g^{2} L_{1}}{4}(r \cos \theta)^{2}}}{(r \cos \theta)^{2}}=4 \pi^{3} \frac{\mathrm{e}^{-\frac{g^{2} L_{1}}{4} r^{2}}}{r^{2}}
\end{gathered}
$$

where $\cos \theta=\cos \theta_{2} \cos \theta_{1}+\sin \theta_{2} \sin \theta_{1} \cos \left(\phi_{2}-\phi_{1}\right)$

Thus, the magnetization density function and the electric charge density function of a proton are represented by

$$
\begin{gathered}
\rho_{m}^{[P]}=\left(d_{1} m\right)^{2} 4 \pi\left[\left(1+3 \pi^{2}\right) \frac{\mathrm{e}^{-\frac{g^{2} L_{1}}{4} r^{2}}}{r^{2}}\right] \\
\rho_{c h}^{[P]}=\left(d_{2} e\right)^{2} 4 \pi\left[2 \frac{\mathrm{e}^{-\frac{g^{2} L_{1} r^{2}}{r^{2}}} \exp \left(-\left(\frac{r^{2}}{m_{P}}\right)^{n_{P}}\right)}{+}\right. \\
\left.+\left[\left(1+3 \pi^{2}\right) \frac{\mathrm{e}^{-\frac{g^{2} L_{1}}{4}(r+\beta)^{2}}}{(r+\beta)^{2}}\right]\left(1-\exp \left(-\left(\frac{r^{2}}{m_{P}}\right)^{n_{P}}\right)\right)\right]
\end{gathered}
$$

In Equation (35), the $\exp \left(-\left(\frac{r^{2}}{m_{P}}\right)^{n_{P}}\right)$ term shows at what radius asymptotic freedom begins and we treat the $m_{P}, n_{P}$ and $\beta$ values as parameters.

For a neutron, we consider that it is constructed of a $\pi^{+}-\pi^{-}$pair as mentioned in sec. 2-1. Because $\pi^{-}$is an antiparticle of $\pi^{+}$, the WF of $\pi^{-}$can be considered to be the same as that of $\pi^{+}$. Therefore, the basis of the electric charge density function of a neutron is represented as

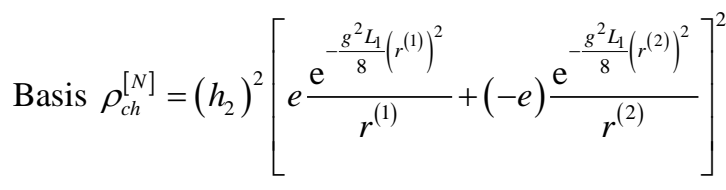

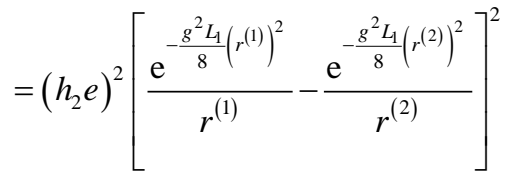


Using the same consideration for the vectors $\vec{r}^{(1)}$ and $\vec{r}^{(2)}$ as that of a proton, the basis of the electric charge density function becomes

Basis $\rho_{c h}^{[N]}=\left(h_{2} e\right)^{2}\left[4 \pi \frac{\mathrm{e}^{-\frac{g^{2} L_{1}}{4} r^{2}}}{r^{2}}-\int_{0}^{\pi} \mathrm{d} \theta_{2} \int_{0}^{2 \pi} \mathrm{d} \phi_{2} \int_{0}^{\pi} \sin \theta_{1} \int_{0}^{2 \pi} \mathrm{d} \phi_{1}\left(2 \frac{\mathrm{e}^{-\frac{g^{2} L_{1}}{8} r^{2}\left(1+(\cos \theta)^{2}\right)}}{r^{2} \cos \theta}-\frac{\mathrm{e}^{-\frac{g^{2} L_{1}}{4}(r \cos \theta)^{2}}}{(r \cos \theta)^{2}}\right)\right]$

$=\left(h_{2} e\right)^{2} 4 \pi\left[\left(1-\pi^{2}\right) \frac{\mathrm{e}^{-\frac{g^{2} L_{1}}{4} r^{2}}}{r^{2}}\right]$

For the magnetization density function of a neutron $\rho_{m}^{[N]}$, the form is the same as the basis of the electric charge density function, but positive. Then $\rho_{m}^{[\mathrm{N}]}$ is represented as

$$
\rho_{m}^{[N]}=\left(h_{1} m\right)^{2} 4 \pi\left[\left(\pi^{2}-1\right) \frac{\mathrm{e}^{-\frac{g^{2} L_{1}}{4} r^{2}}}{r^{2}}\right]
$$

For the electric charge density function of neutron, we again consider asymptotic freedom. At large $r$ (small $|\vec{Q}|^{2}$ ), they move with the same origin, but, at small $r$ (large $|\vec{Q}|^{2}$ ), $\pi^{+}$and $\pi^{-}$move independently.

To be precise,

3) as $r \rightarrow 0$,

$$
\rho_{c h}^{[N]} \rightarrow\left(h_{2} e\right)^{2}(4 \pi) 2 \frac{\mathrm{e}^{-\frac{g^{2} L_{1} r^{2}}{4}}}{r^{2}}
$$

4) as $r \rightarrow \infty$,

$$
\rho_{c h}^{[N]} \rightarrow\left(h_{2} e\right)^{2} 4 \pi\left[\left(1-\pi^{2}\right) \frac{\mathrm{e}^{-\frac{g^{2} L_{1}}{4} r^{2}}}{r^{2}}\right]
$$

Using the same expression resulting from asymptotic freedom for the proton case, $\rho_{c h}^{[N]}$ is represented by

$$
\begin{aligned}
\rho_{c h}^{[N]}= & \left(h_{2} e\right)^{2} 4 \pi\left[2 \frac{\mathrm{e}^{-\frac{g^{2} L_{1}}{4} r^{2}}}{r^{2}} \exp \left(-\left(\frac{r^{2}}{m_{N}}\right)^{n_{N}}\right)\right. \\
& \left.+\left[\left(1-\pi^{2}\right) \frac{\mathrm{e}^{-\frac{g^{2} L_{1}}{4}(r+\beta)^{2}}}{(r+\beta)^{2}}\right]\left(1-\exp \left(-\left(\frac{r^{2}}{m_{N}}\right)^{n_{N}}\right)\right)\right]
\end{aligned}
$$

where $m_{N}, n_{N}$ and $\beta$ are parameters.

\subsubsection{Form Factors}

To evaluate the Sachs e.m. FFs of protons and neutrons, i.e., $G_{E}^{[P]}, G_{M}^{[P]}, G_{E}^{[N]}$ and $G_{M}^{[N]}$, we adopt the following relations proposed by Mitra and Kumari [29]. 
Accordingly, intrinsic FFs $\tilde{\rho}_{m}(k)$ and $\tilde{\rho}_{c h}(k)$ are related to the Sachs e.m. FFs $G_{M}\left(|\vec{Q}|^{2}\right)$ and $G_{E}\left(|\vec{Q}|^{2}\right)$ as

$$
\begin{gathered}
\tilde{\rho}_{c h}(k)=(1+\tau)^{2} G_{E}\left(|\vec{Q}|^{2}\right) \\
\mu_{(i)} \tilde{\rho}_{m}(k)=(1+\tau)^{2} G_{M}\left(|\vec{Q}|^{2}\right)
\end{gathered}
$$

where $\tau=\frac{|\vec{Q}|^{2}}{(2 M)^{2}}, i=P$ or $N\left(\mu_{P}\right.$ and $\mu_{N}$ are the magnetic moment of a proton and a neutron, respectively).

and $\tilde{\rho}(k) s$ are the Fourier transform of the electric charge and magnetization density functions of a nucleon.

Under relativistic consideration, the relationship between $k^{2}$ and $|\vec{Q}|^{2}$ is

$$
k^{2}=|\vec{q}|^{2} \rightarrow \frac{|\vec{Q}|^{2}}{1+\tau}
$$

and for the nonrelativistic case, the relationship between $k=|\vec{q}|$ and $|\vec{Q}|$ is $k=|\vec{q}| \rightarrow|\vec{Q}|$.

We derived the electric charge and magnetization density functions in sec 2-3 (a) so that in principle, we only need to take the Fourier Transforms to obtain the Sachs e.m. FFs.

For the magnetization density functions, we can use the Fourier transform directly. However, for the electric charge density functions, we cannot use the exact transformations because that the rigorous Fourier transform cannot reflect the asymptotic freedom characteristics in momentum space. Thus, in the electric charge density function case, we take the Fourier transform of the basis of the electric charge density functions and express the asymptotic freedom in momentum space by adopting a description similar to that used in the configuration space. We then use the relation of Equation (42) to obtain the Sachs FFs of $G_{E}$. The electric charge density functions of protons and neutrons were given in sec. 2-3 (a) as

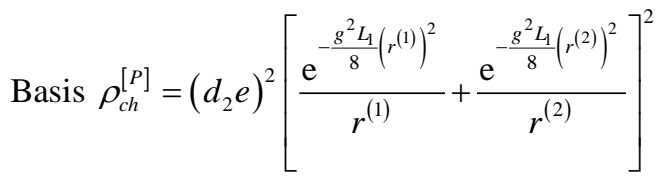

$$
\begin{aligned}
& \text { Basis } \rho_{c h}^{[N]}=\left(h_{2} e\right)^{2}\left[\frac{\mathrm{e}^{-\frac{g^{2} L_{1}\left(r^{(1)}\right)^{2}}{8}}}{r^{(1)}}-\frac{\mathrm{e}^{-\frac{g^{2} L_{1}}{8}\left(r^{(2)}\right)^{2}}}{r^{(2)}}\right]^{2}
\end{aligned}
$$

Note that, other than the proportional constants, the only difference between Basis $\rho_{c h}^{[P]}$ and Basis $\rho_{c h}^{[N]}$ is the sign.

Considering again the direction of longitudinal momentum $\vec{Q}$ to be the $\mathrm{z}$ 
axis and considering the polar coordinates $\vec{r}^{(1)}=\left(r^{(1)}, \theta_{1}, \phi_{1}\right)$ and $\vec{r}^{(2)}=\left(r^{(2)}, \theta_{2}, \phi_{2}\right)$, and again considering $\vec{r}^{(1)}$ as $\vec{r}$, the Fourier transform of Basis $\rho_{c h}^{[P]}$ and Basis $\rho_{c h}^{[N]}$ can be expressed as

$$
\begin{aligned}
& \text { Basis } \tilde{\rho}_{c h}^{[P, N]}(|\vec{q}|=k) \\
& =\text { const } 2 \pi \int_{0}^{\infty} r^{2} \mathrm{~d} r \int_{0}^{\pi} \sin \theta_{1} \mathrm{~d} \theta_{1} \mathrm{e}^{-i|\vec{q}| r \cos \theta_{1}} \\
& \times\left[\mp \frac{\mathrm{e}^{-\frac{g^{2} L_{1}}{4} r^{2}}}{r^{2}}+\int_{0}^{\pi} \mathrm{d} \theta_{2} \int_{0}^{2 \pi} \mathrm{d} \phi\left(2 \frac{\mathrm{e}^{-\frac{g^{2} L_{1}}{8} r^{2}\left(1+(\cos \theta)^{2}\right)}}{r^{2} \cos \theta} \mp \frac{\mathrm{e}^{-\frac{g^{2} L_{1}}{4}(r \cos \theta)^{2}}}{(r \cos \theta)^{2}}\right)\right]
\end{aligned}
$$

where $\cos \theta=\cos \theta_{2} \cos \theta_{1}+\sin \theta_{2} \sin \theta_{1} \cos \left(\phi_{2}-\phi_{1}\right)$.

For the second line, we take $\phi_{2}-\phi_{1}=\phi$.

The first term of Equation (46) becomes

$$
\begin{aligned}
\text { First term } & =\text { const } 2 \pi \int_{0}^{\infty} r^{2} \mathrm{~d} r \int_{0}^{\pi} \sin \theta_{1} \mathrm{~d} \theta_{1} \mathrm{e}^{-i|\overrightarrow{\mid}| \cos \theta_{1}} \frac{\mathrm{e}^{-\frac{g^{2} L_{1} r^{2}}{r^{2}}}}{2 \sqrt{g^{2} L_{1}}} \exp \left(-\frac{|q|^{2}}{g^{2} L_{1}}\right) F\left(1 ; \frac{3}{2} ; \frac{|\vec{q}|^{2}}{g^{2} L_{1}}\right) \\
& =\operatorname{const} 4 \pi \frac{\sqrt{\pi}}{}=\operatorname{const} 2 \pi \frac{\sqrt{\pi}}{\sqrt{g^{2} L_{1}}} F_{\pi}
\end{aligned}
$$

where $F(\alpha ; \beta ; z)$ is the Kummer's confluent hypergeometric series.

We showed this integral result previously [26].

The second and third terms of Equation (46) become

Fourier cosine of the second term

$$
\begin{aligned}
& =2 \operatorname{const} \int_{0}^{\pi} \mathrm{d} \theta_{2} \int_{0}^{2 \pi} \mathrm{d} \phi 2 \pi \int_{0}^{\infty} r^{2} \mathrm{~d} r \int_{0}^{\pi} \sin \theta_{1} \mathrm{~d} \theta_{1} \cos \left(|\vec{q}| r \cos \theta_{1}\right) \frac{\mathrm{e}^{-\frac{g^{2} L_{1}}{8} r^{2}\left(1+(\cos \theta)^{2}\right)}}{r^{2} \cos \theta} \\
& =2 \operatorname{const} \pi \frac{2 \pi^{2} \sqrt{\pi}}{\sqrt{g^{2} L_{1}}} \exp \left(-\frac{|\vec{q}|^{2}}{g^{2} L_{1}}\right)
\end{aligned}
$$

Fourier sine of second term

$$
\begin{aligned}
& =2 \operatorname{const} \int_{0}^{\pi} \mathrm{d} \theta_{2} \int_{0}^{2 \pi} \mathrm{d} \phi 2 \pi \int_{0}^{\infty} r^{2} \mathrm{~d} r \int_{0}^{\pi} \sin \theta_{1} \mathrm{~d} \theta_{1} \sin \left(|\vec{q}| r \cos \theta_{1}\right) \frac{\mathrm{e}^{-\frac{g^{2} L_{1}}{8} r^{2}\left(1+(\cos \theta)^{2}\right)}}{r^{2} \cos \theta} \\
& =2 \operatorname{cons} \pi \frac{4 \pi^{2}}{g^{2} L_{1}} \sqrt{|\vec{q}|^{2}} \exp \left(-\frac{|\vec{q}|^{2}}{g^{2} L_{1}}\right) F\left(\frac{1}{2} ; \frac{3}{2} ; \frac{|\vec{q}|^{2}}{g^{2} L_{1}}\right)
\end{aligned}
$$

Fourier cosine of the third term

$$
\begin{aligned}
& =\text { const } \int_{0}^{\pi} \mathrm{d} \theta_{2} \int_{0}^{2 \pi} \mathrm{d} \phi 2 \pi \int_{0}^{\infty} r^{2} \mathrm{~d} r \int_{0}^{\pi} \sin \theta_{1} \mathrm{~d} \theta_{1} \cos \left(|\vec{q}| r \cos \theta_{1}\right) \frac{\mathrm{e}^{-\frac{g^{2} L_{1}}{4} r^{2}(\cos \theta)^{2}}}{(r \cos \theta)^{2}} \\
& =2 \operatorname{const} \pi \frac{2 \pi^{2} \sqrt{\pi}}{\sqrt{g^{2} L_{1}}} \exp \left(-\frac{|\vec{q}|^{2}}{g^{2} L_{1}}\right)
\end{aligned}
$$




$$
\text { Fourier sine og third term }=0
$$

Therefore, the Fourier transform of the basis of the electric charge density function of protons and neutrons becomes

$$
\begin{aligned}
& \text { Basis } \tilde{\rho}_{c h}^{[P]}(|\vec{q}|) \\
& =\operatorname{const} 2 \pi \frac{\sqrt{\pi}}{\sqrt{g^{2} L_{1}}}\left[F_{\pi}+4 \frac{\pi^{\frac{3}{2}}}{\sqrt{g^{2} L_{1}}} \sqrt{|\vec{q}|^{2}} \exp \left(-\frac{|\vec{q}|^{2}}{g^{2} L_{1}}\right) F\left(\frac{1}{2} ; \frac{3}{2} ; \frac{|\vec{q}|^{2}}{g^{2} L_{1}}\right)\right. \\
& +p \frac{1}{|\vec{Q}|}\left[\log \sqrt{\frac{2|\vec{q}|^{2}}{g^{2} L_{1}}}-\exp \left(-\sqrt{\frac{2|\vec{q}|^{2}}{g^{2} L_{1}}}\right) \sum_{n=1}^{\infty} \frac{1}{n !}\left(\sum_{r=1}^{n} \frac{1}{r}\right)\left(\sqrt{\frac{2|\vec{q}|^{2}}{g^{2} L_{1}}}\right)^{n}\right] \\
& \left.+4 \pi^{2} \exp \left(-\frac{|\vec{q}|^{2}}{g^{2} L_{1}}\right)\right]
\end{aligned}
$$

Basis $\tilde{\rho}_{c h}^{[N]}(|\vec{q}|)$

$$
\begin{aligned}
= & \text { const } 2 \pi \frac{\sqrt{\pi}}{\sqrt{g^{2} L_{1}}} \mid-F_{\pi}+4 \frac{\pi^{\frac{3}{2}}}{\sqrt{g^{2} L_{1}}} \sqrt{|\vec{q}|^{2}} \exp \left(-\frac{|\vec{q}|^{2}}{g^{2} L_{1}}\right) F\left(\frac{1}{2} ; \frac{3}{2} ; \frac{|\vec{q}|^{2}}{g^{2} L_{1}}\right) \\
& +p \frac{1}{|\vec{q}|}\left[\log \sqrt{\frac{2|\vec{q}|^{2}}{g^{2} L_{1}}}-\exp \left(-\sqrt{\frac{2|\vec{q}|^{2}}{g^{2} L_{1}}}\right) \sum_{n=1}^{\infty} \frac{1}{n !}\left(\sum_{r=1}^{n} \frac{1}{r}\right)\left(\sqrt{\frac{2|\vec{q}|^{2}}{g^{2} L_{1}}}\right)^{n}\right] \\
& +4 \pi^{2} \exp \left(-\frac{|\vec{q}|^{2}}{g^{2} L_{1}}\right)
\end{aligned}
$$

Thus,

$$
\begin{aligned}
& \text { Basis } \tilde{\rho}_{c h}^{[P, N]}(|\vec{q}|) \\
& =\text { const } 2 \pi \frac{\sqrt{\pi}}{\sqrt{g^{2} L_{1}}}\left[\mp F_{\pi}+4 \frac{\pi^{\frac{3}{2}}}{\sqrt{g^{2} L_{1}}} \sqrt{|\vec{q}|^{2}} \exp \left(-\frac{|\vec{q}|^{2}}{g^{2} L_{1}}\right) F\left(\frac{1}{2} ; \frac{3}{2} ; \frac{|\vec{q}|^{2}}{g^{2} L_{1}}\right)\right. \\
& +p \frac{1}{|\vec{q}|}\left[\log \sqrt{\frac{2|\vec{q}|^{2}}{g^{2} L_{1}}}-\exp \left(-\sqrt{\frac{2|\vec{q}|^{2}}{g^{2} L_{1}}}\right) \sum_{n=1}^{\infty} \frac{1}{n !}\left(\sum_{r=1}^{n} \frac{1}{r}\right)\left(\sqrt{\frac{2|\vec{q}|^{2}}{g^{2} L_{1}}}\right)^{n}\right] \\
& \left.+4 \pi^{2} \exp \left(-\frac{|\vec{q}|^{2}}{g^{2} L_{1}}\right)\right]
\end{aligned}
$$

where $p$ is parameter.

Ye et al. used relativistic considerations for their parametrization work [3]. However, in our case, we use nonrelativistic consideration, in which $k=|\vec{q}|$ simply relates to $|\vec{Q}|$. According to Kelly [22], if one knew how to obtain an intrinsic form factor $\tilde{\rho}(k)$ from data for appropriate Sachs form factor, the intrinsic density could be obtained simply by inverting Fourier transform and the naïve nonrelativistic inversion method assumes that $k \rightarrow Q$ and $\tilde{\rho}(k) \rightarrow G\left(Q^{2}\right)$ where $G\left(Q^{2}\right)$ is the appropriate Sachs form factor. However, 
the reason why the nonrelativistic inversion was abandoned is that it produces unsatisfactory results. The corresponding radial densities have an unphysical cusp at the origin and rather hard cores. However, even using relativistic inversin method, this has unphysical failor. Again according to Kelly [22], it is that unique relativistic relationship between the Sachs form factors measured by electron scattering at finite $Q^{2}$ and the static charge magnetization densities in nucleon rest frame do not exsit. The basic problem is that electron scattering measures transition matrix elements between state of a composite system that have different momenta and the transition densities between such states are different from the static densities in the rest frame. Firthermore, the boost operator for a composite system depends upon the interaction among its constituents. On the other hand, for our case, we consider that a nuleon is described as a pair of pions which means that the WF has a singularity at the origin. The appearance of a cusp at the origin is rather satisfacotry and is our reason for choosing the nonrelativistic inversion. Thus, from now on, we simply replace $|\vec{q}|$ with $|\vec{Q}|$. To construct the electric charge density functions of protons and neutrons in momentum space, we again consider the asymptotic freedom description, just as we did for configuration space. That is, for the small $|\vec{Q}|^{2}$ case, the pair of pions moves with respect to the same origin. However, as $|\vec{Q}|^{2}$ becomes larger, the two pions begin to move separately and finally move totally independent of each other.

To be precise, this situation is described as

$$
\begin{gathered}
\tilde{\rho}_{c h}^{[P]}(|\vec{Q}|) \\
=\operatorname{const} 2 \pi \frac{\sqrt{\pi}}{\sqrt{g^{2} L_{1}}} F_{\pi}\left(1+\left(\left(z_{1}+z_{2}+z_{3}\right) / F_{\pi}\right) /\left(\exp \left(-\left(\frac{|\vec{Q}|^{2}}{m_{P}}\right)^{n_{P}}\right)\right.\right. \\
\left.+\left(1-\exp \left(-\left(\frac{|\vec{Q}|^{2}}{m_{P}}\right)^{n_{P}}\right)\right)\left(\frac{z_{1}+z_{2}+z_{3}}{F_{\pi}}\right)\right) \\
\tilde{\rho}_{c h}^{[N]}(|\vec{Q}|) \\
=\operatorname{const~} 2 \pi \frac{\pi^{\frac{3}{2}}}{\sqrt{g^{2} L_{1}}} F_{\pi}\left(1-z_{3} / F_{\pi}+\left(\frac{\left(z_{1}+z_{2}+z_{3}\right)}{F_{\pi}}-1\right) /\left(\exp \left(-\left(\frac{|\vec{Q}|^{2}}{m_{N}}\right)^{n_{N}}\right)\right.\right. \\
\left.+\left(1-\exp \left(-\left(\frac{|\vec{Q}|^{2}}{m_{N}}\right)^{n_{N}}\right)\right)\left(\left(\frac{\left(z_{1}+z_{2}+z_{3}\right)}{F_{\pi}}-1\right)\right)\right)
\end{gathered}
$$

where

$$
z_{1}=4 \frac{\pi^{\frac{3}{2}}}{\sqrt{g^{2} L_{1}}} \sqrt{|\vec{Q}|^{2}} \exp \left(-\frac{|\vec{Q}|^{2}}{g^{2} L_{1}}\right) F\left(\frac{1}{2} ; \frac{3}{2} ; \frac{|\vec{Q}|^{2}}{g^{2} L_{1}}\right)
$$




$$
\begin{gathered}
z_{2}=p \frac{1}{\sqrt{|\vec{Q}|^{2}}}\left[\log \sqrt{\frac{2|\vec{Q}|^{2}}{g^{2} L_{1}}}-\exp \left(-\sqrt{\frac{2|\vec{Q}|^{2}}{g^{2} L_{1}}} \sum_{n=1}^{\infty} \frac{1}{n !}\left(\sum_{r=1}^{n} \frac{1}{r}\right)\left(\sqrt{\frac{2|\vec{Q}|^{2}}{g^{2} L_{1}}}\right)^{n}\right]\right. \\
z_{3}=4 \pi^{2} \exp \left(-\frac{|\vec{Q}|^{2}}{g^{2} L_{1}}\right)
\end{gathered}
$$

where $p$ of $z_{2}$ is parameter.

These expressions are not exactly Fourier transforms of the electric charge density functions. However, because the basis of $\tilde{\rho}_{c h}(k)$ is exactly the Fourier transform of the basis of $\rho_{c h}(r)$, we use the relationship between Sachs e.m. FFs and intrinsic FFs shown in Equation (42) and Equation (43) to obtain in the Sachs e.m. FFs as

$$
\begin{aligned}
G_{E}^{[P]}= & \frac{1}{(1+\tau)^{2}} F_{\pi}\left(1+\left(\left(z_{1}+z_{2}+z_{3}\right) / F_{\pi}\right) /\left(\exp \left(-\frac{|\vec{Q}|^{2}}{m_{P}}\right)^{n_{P}}\right)\right. \\
& \left.\left.+\left(1-\exp \left(-\left(\frac{|\vec{Q}|^{2}}{m_{P}}\right)^{n_{P}}\right)\right) \frac{z_{1}+z_{2}+z_{3}}{F_{\pi}}\right)\right) \\
G_{E}^{[N]}= & \frac{1}{(1+\tau)^{2}} F_{\pi}\left(1-z_{3} / F_{\pi}+\left(\frac{\left(z_{1}+z_{2}+z_{3}\right)}{F_{\pi}}-1\right) /\left(\exp \left(-\left(\frac{|\vec{Q}|^{2}}{m_{N}}\right)^{n_{N}}\right)\right.\right. \\
& \left.\left.+\left(1-\exp \left(-\left(\frac{|\vec{Q}|^{2}}{m_{N}}\right)^{n_{N}}\right)\right)\left(\frac{\left(z_{1}+z_{2}+z_{3}\right)}{F_{\pi}}-1\right)\right)\right)
\end{aligned}
$$

where $z_{1}, z_{2}, z_{3}$ are given in Equation (57) to Equation (59), and $\tau$ is given as

$$
\tau=\frac{|\vec{Q}|^{2}}{(2 M)^{2}}
$$

here, $M$ is the characteristic mass and it is taken as a parameter.

The relationships between the magnetization density functions and the Sachs e.m. FFs, i.e., $G_{M}^{[P]}, G_{M}^{[N]}$, are exactly formulated by their Fourier transform using Equation (43).

Then, we obtain

$$
\begin{aligned}
& G_{M}^{[P]}=\frac{1}{(1+\tau)^{2}}\left(F_{\pi}\left[1+\left(z_{1}+z_{2}+z_{3}\right) / F_{\pi}\right]\right) \\
& G_{M}^{[N]}=\frac{1}{(1+\tau)^{2}} F_{\pi}\left[-1+\left(z_{1}+z_{2}+z_{3}\right) / F_{\pi}\right]
\end{aligned}
$$

where $\tau$ is the same as Equation (59) and $z_{1}, z_{2}$ and $z_{3}$ are given in Equa- 
tion (57), Equation (58), and Equation (59).

Note that our Sachs e.m. FFs have normalization uncertainty. To compare our values with the parametrization results of Ye et al. [3], we have to normalize our $G_{M}^{[P, N]}$ and $G_{E}^{[P, N]}$ by dividing them by some constant values. These normalization constants are chosen to be comparable to the above parametrization results. Then the normalized $G_{M}^{[P]}$ and $G_{M}^{[N]}$ correspond to the normal $G_{M}^{[P]} / \mu_{P}$ and $G_{M}^{[N]} / \mu_{N}$, respectively. In section 3 and Figures $1-8$ we use $G_{M}^{[P]}$ and $G_{M}^{[N]}$ to denote $G_{M}^{[P]} / \mu_{P}$ and $G_{M}^{[N]} / \mu_{N}$, respectively.

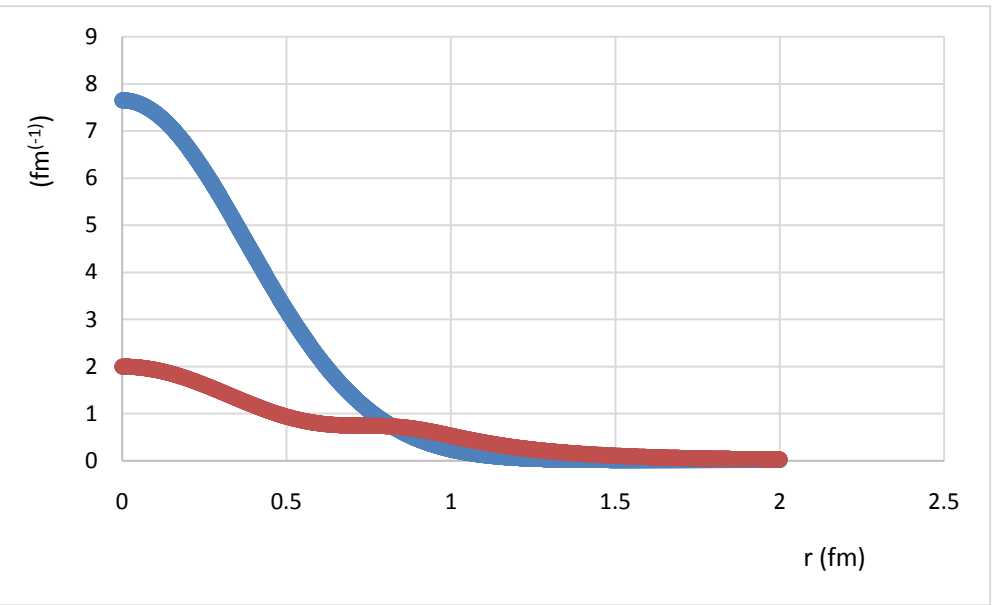

Figure 1. Proton magnetization and electric charge density functions. 1) Blue line is proton magnetization density function (multiplied by $r^{2}$ ) $r^{2} \rho_{m a g}^{[P]}$ (magnitude is $\frac{1}{2}$ );2) Orange line is proton electric charge density function (multiplied by $r^{2}$ ) $r^{2} \rho_{c h}^{[P]} . n_{p}=4 ; m_{p}=0.7 ; \beta=0.1$.

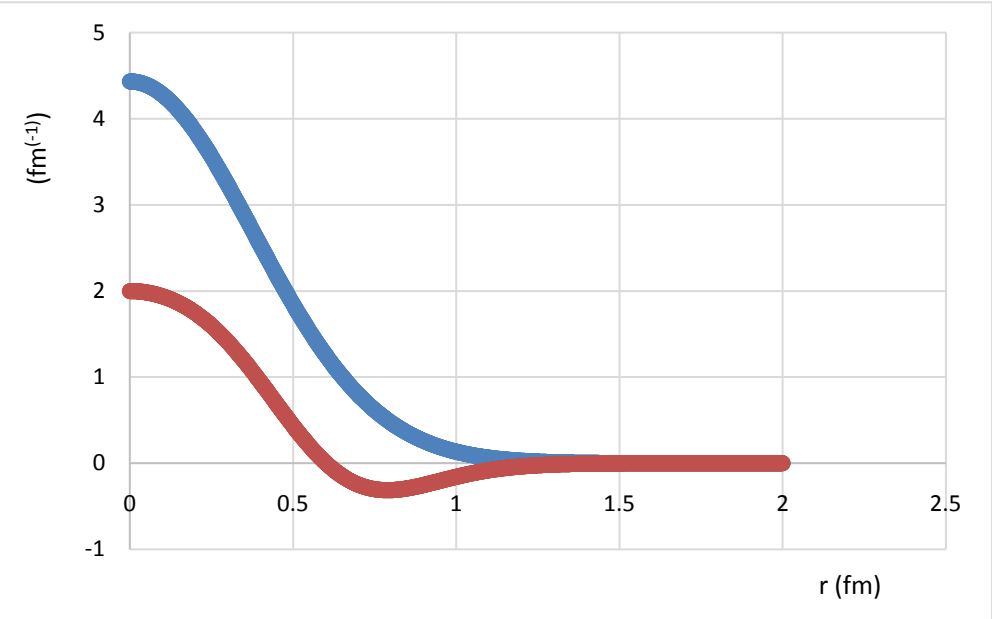

Figure 2. Neutron magnetization and electric charge density function. 1) Blue line is neutron magnetization density function (multiplied by $r^{2}$ ) $\left.r^{2} \rho_{m a g}^{[N]} ; 2\right)$ Orange line is neutron electric charge density function (multiplied by $\left.r^{2}\right) r^{2} \rho_{c h}^{[N]} . n_{N}=4 ; \quad m_{N}=0.8 ; \quad \beta=0.05$. 


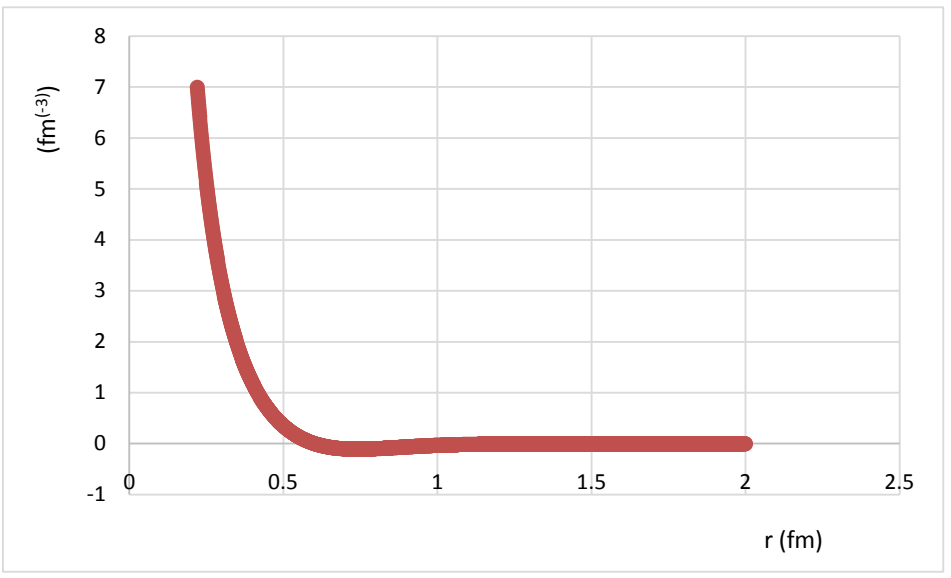

Figure 3. Electric charge density function of neutron $\rho_{c h}^{[N]}$. Note that this becomes negative values (refer Figure 2) beyond $0.6 \mathrm{fm}$ region as same as those of Galster model and Maints data beyond $0.7 \mathrm{fm}$ region and that the factor of $1 / 5$ is multiplied.

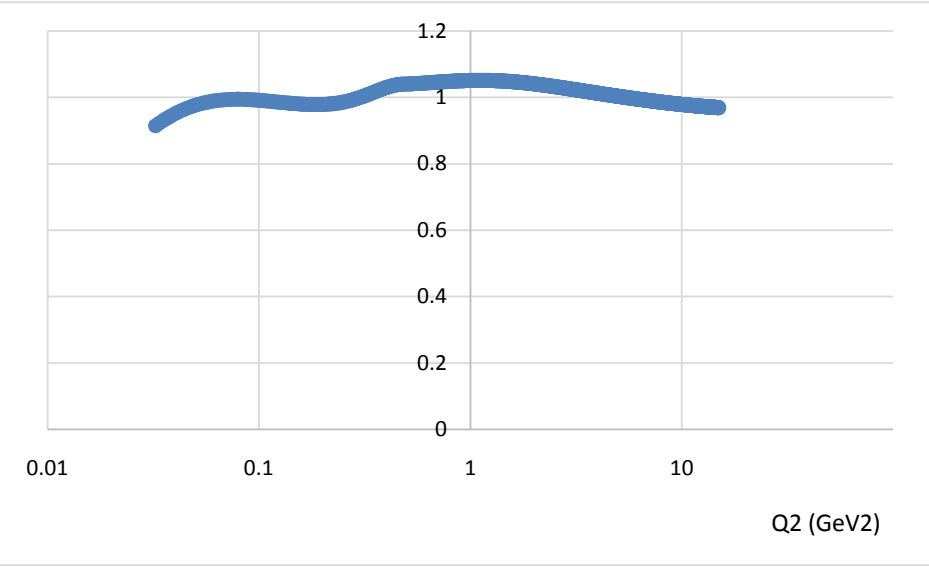

Figure 4. Sachs proton magnetization form factor (divided by $G_{D}$ ) $G_{M}^{[P]} / G_{D}$. Parameter $p: p=3.6284$.

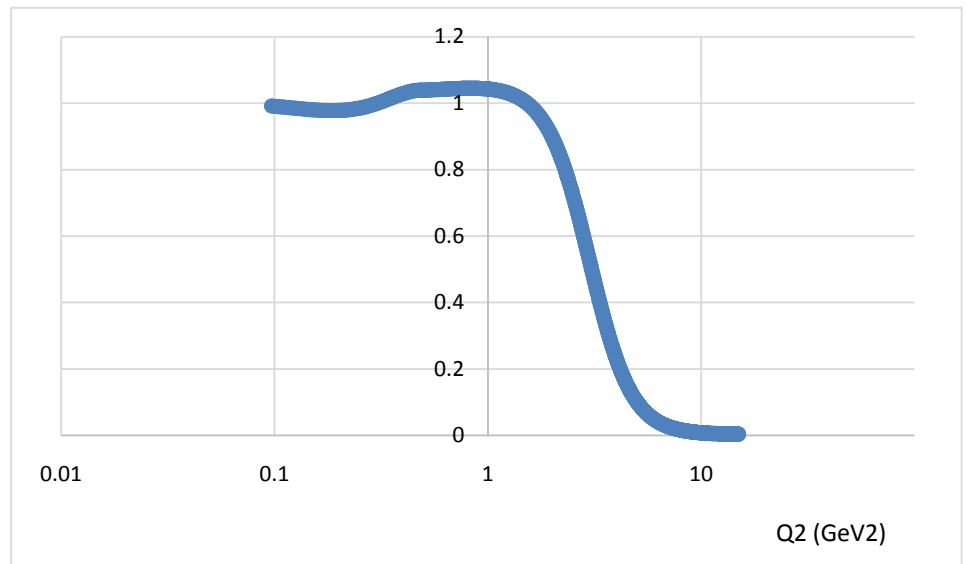

Figure 5. Sachs proton electric charge form factor (divided by $G_{D}$ ) $G_{E}^{[P]} / G_{D} \cdot n_{E}^{[P]}=4 ; \quad m_{E}^{[P]}=12 ; p=3.6284$. 


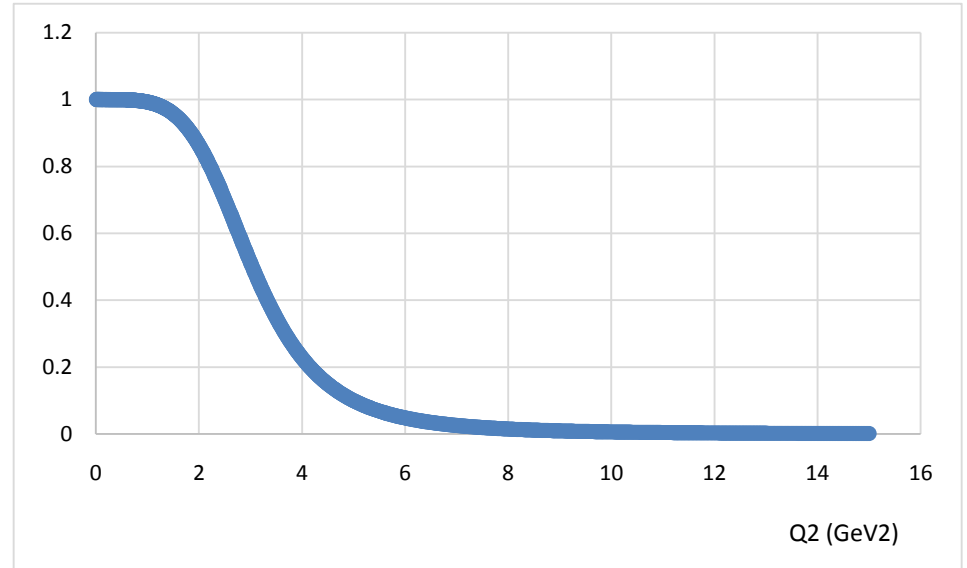

Figure 6. Ratio of $G_{E}^{[P]} / G_{M}^{[P]}$.

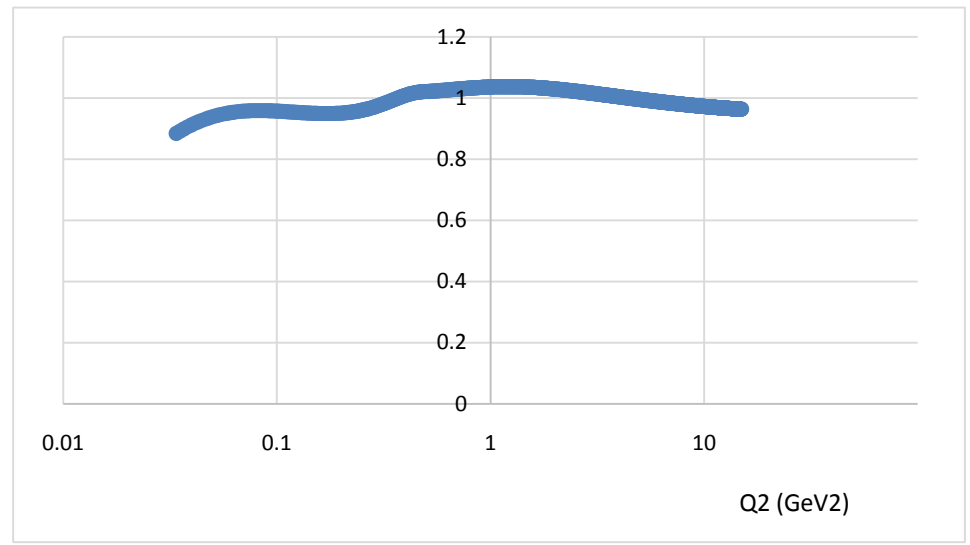

Figure 7. Sachs neutron magnetization form factor (divided by $G_{D}$ ) $G_{M}^{[N]} / G_{D}$. Parameter $p: p=3.6284$.

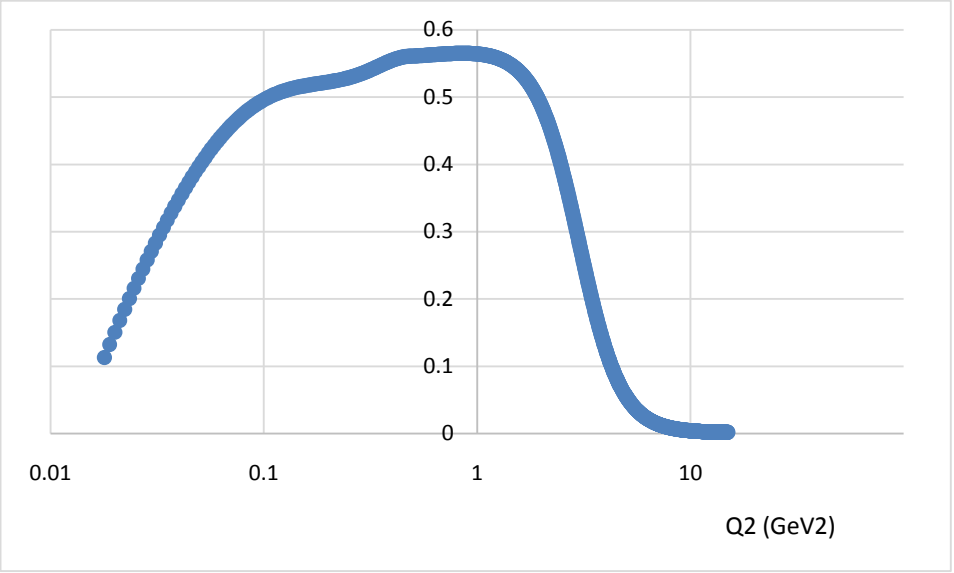

Figure 8. Sachs neutron electric charge form factor (divided by $G_{D}$ ) $G_{E}^{[N]} / G_{D} \cdot n_{E}^{[N]}=4 ; \quad m_{E}^{[N]}=12 ; p=3.6284$.

\section{Results}

Using Equation (34), Equation (35), Equation (38) and Equation (41), we show 
the magnetization and electric charge density functions of protons and neutrons in Figure 1 and Figure 2, respectively. Note that the shown density functions are $r^{2} \rho_{m}^{[P]}, r^{2} \rho_{c h}^{[P]}, r^{2} \rho_{m}^{[N]}$ and $r^{2} \rho_{c h}^{[N]}$ instead of just density functions because of the existence of the singularity at the origin (at $r=0$ ) that results from our definition of the density functions. In addition, we show the electric charge density function of neutron $\rho_{c h}^{[N]}$ in Figure 3.

To confine the sizes of protons and neutrons less than $1.2 \mathrm{fm}$, we chose the Gaussian parameter to be $3.5\left(\mathrm{GeV}^{2}\right)$. Using this value, we obtain the characteristic mass $\sqrt{\frac{g^{2} L_{1}}{2}}$ of $1025(\mathrm{MeV})$, which is similar to the $\phi$ meson mass. This is different from the pion mass of $140 \mathrm{MeV}$ that we use to evaluate Sachs' proton and neutron e.m. FFs later in this paper.

Figure 1 and Figure 2 show that our density functions (multiplied by $r^{2}$ ) do not behave exactly like the density functions of Kelly [22], especially, behavior at near origin.

However, Kelly's density functions were obtained by using the relativistic inversion method, which is adopted for preventing them from showing the cusp at origin. To be clear this point, our electric charge density function of neutron in Figure 3 can be compared to those of Galster model [30] and Mainz data analysed by Schmieden [31]. Their results are shown in Figure 12 of Kelly [22]. Note that their results were obtained using by the nonrelativistic inversion of Fourier transform which is same as our case. Both density functions have singularity at origin although the magnitude of singularity is smaller than ours because their $r^{2} \rho_{c h}^{[N]}$ goes to 0 as $r$ approach 0 . Except the magnitude of singularity, their whole behaviors were quite similar to ours. The density functions of proton also have a similarity such that the proton electric density function overwhelms the proton magnetization density function beyond $0.8 \mathrm{fm}$. To notify this similarity, we compare our $r^{2} \rho_{c h}^{[P]}, r^{2} \rho_{m}^{[P]}$ with $\rho_{c h}^{[P]}, \rho_{m}^{[P]}$ in Figure 5 and Figure 6 of Kelly's [22] because we are focused in the behavior except in near origin region. This phenomena also appears in the proton electric charge and magnetization density functions of Kelly [22].

Using Equation (60) and Equation (63) with appropriate normalization, we show the results of $G_{M}^{[P]} / G_{D}, G_{E}^{[P]} / G_{D}$ and $G_{E}^{[P]} / G_{M}^{[P]}$ in Figures 4-6. Our evaluation forms for Sachs e.m. FFs are not appropriate to show the behavior of the form FFs in the region where $|\vec{Q}|^{2}$ is smaller than $10^{-1}\left(\mathrm{GeV}^{2}\right)$. However, they are sufficiently applicable in the region where $|\vec{Q}|^{2}$ is larger than $10^{-1}$ $\left(\mathrm{GeV}^{2}\right)$. Thus we can compare our results to the parametrization results in Ye et al. [3] in the region where $|\vec{Q}|^{2}$ is larger than $10^{-1}\left(\mathrm{GeV}^{2}\right)$. In particular, we obtain a fairly good result for $G_{E}^{[P]} / G_{M}^{[P]}$ and also it is quite similar to that of CQM by Miller shown in Arrington [34] up to $4\left(\mathrm{GeV}^{2}\right)$ as we expected.

Using Equation (61) and Equation (64) with appropriate normalization, we show the results of $G_{M}^{[N]} / G_{D}, G_{E}^{[N]} / G_{D}$ and $G_{E}^{[N]} / G_{M}^{[N]}$ in Figures 7-9. The magnetization FFs for both protons and neutrons have very similar features to 


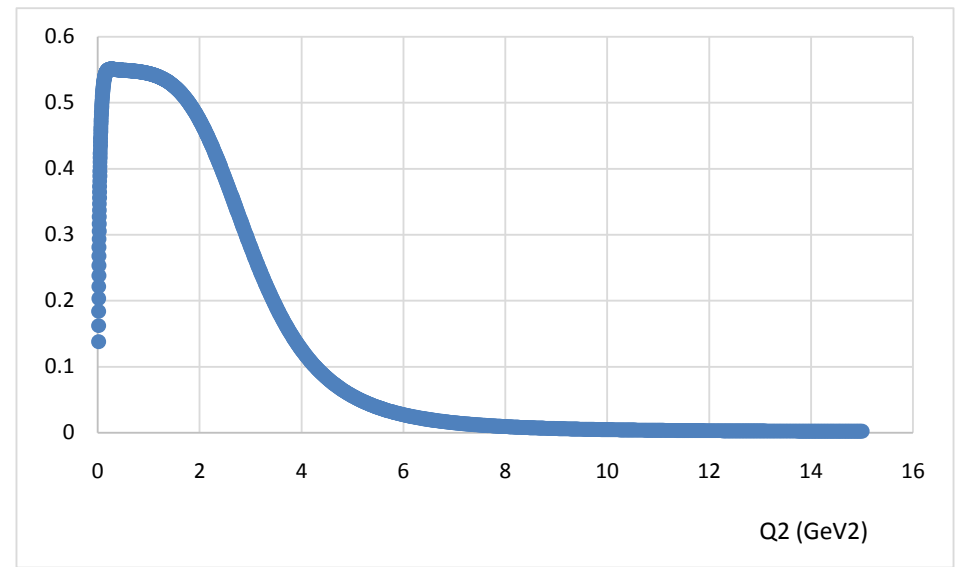

Figure 9. Ratio of $G_{E}^{[N]} / G_{M}^{[N]}$.

those resulting from parametrization. However, the values of $G_{E}^{[N]} / G_{D}$ in the region of $10^{-1}$ to $10^{0}\left(\mathrm{GeV}^{2}\right)$ are larger than those from the parametrization. Thus, our $G_{E}^{[N]} / G_{M}^{[N]}$ shows a faster rising form than it does in other studies [32] [33] [34]. However, the point where our $G_{E}^{[N]} / G_{M}^{[N]}$ is the most different is when it converges to zero. This behavior was proposed by Arrington [34], although a reason was not given.

\section{Conclusion}

We investigate the proton and neutron electromagnetic form factors where the consideration of that nucleon is described as a pion pair. We obtain a good agreement of the electric density function of neutron with Galster model and Maints data except the magnitude of singularity. The density functions of proton also show a similarity to those of Kelly's except near origin. In the case of Sachs e.m. FFs, we obtain a fary good agreement with the parametrization results in Ye et al. Therefore, we consider that our description of a nucleon as a pion pair is one of the meaningful aspects.

\section{Discussion}

As mentioned in conclusion section, we obtain fairy good results in both density functions and Sachs e.m. FFs, however, there is an ambiguous point in our treatment. We do not exactly know the reason why the density functions and the form factors for the magnetization case do not change the form when two pions move independently each other with asymptotic freedom. This may occur because the magnetization arises not as a result of charge distribution, but because of current or spin. Thomas [35] suggests that nucleon spin comes from the orbital angular momentum of $u(\bar{u})$ and $d(\bar{d})$ quarks (antiquarks). For our case, we describe the nucleon as a pion pair, so we can consider the orbital angular momentum of the $u(d)$ quark and the $\bar{d}(\bar{u})$ antiquark; in the case where the pair of pions has the same origin, angular momentum can arise from movement around the origin. For the independently moving case, the pair of pions move 
relatively around the center of mass so that the angular momentum can be considered from movement around the center of mass. The important point is that the nucleon spin has a decisive quantity of $1 / 2$ even though the specific orbits of quarks (antiquarks) cannot be determined. Thus, for the case where the magnetization arises because of spin, the magnetization is not affected by the situation of the pair of pions. Conversely, for the case where the magnetization results from current, current arises from the movement of charges, that is, movement of the $u(d)$ quark and the $\bar{d}(\bar{u})$ antiquark, i.e., movement of the pair of pions. Hence we can consider the same argument as the spin case because the magnetization is characterized by the derivative of magnetic energy with respect to the absolute value of the magnetic field at the origin or the center of mass. Either way, we can say that the situation regarding the pair of pions does not affect the magnetization density, but this is not confirmative. To elucidate this point, we need further investigation.

\section{Conflicts of Interest}

The author declares no conflicts of interest regarding the publication of this paper.

\section{References}

[1] Perdrisat, C.F., Punjabi, V. and Vanderhaeghen, M. (2007) Progress in Particle and Nuclear Physics, 59, 694. https://doi.org/10.1016/j.ppnp.2007.05.001

[2] Arrington, J., de jager, K. and Perdrisat, C.F. (2011) Journal of Physics: Conference Series, 299, Article ID: 012002. https://doi.org/10.1088/1742-6596/299/1/012002

[3] Ye, Z., Arrington, J., Hill, R.J. and Lee, G. (2018) Physics Letters B, 777, 8. https://doi.org/10.1016/j.physletb.2017.11.023

[4] Chung, P.L. and Coester, F. (1991) Physical Review D, 44, 229. https://doi.org/10.1103/PhysRevD.44.229

[5] Schlumpf, F. (1993) Journal of Physics G, 20, 237 (Brief Report) (Relativistic Treatment).

[6] Frank, M.R., Jennings, B.K. and Miller, G.A. (1996) Physical Review C, 54, 920. https://doi.org/10.1103/PhysRevC.54.920

[7] Miller, G.A. and Frank, M.R. (2002) Physical Review C, 65, Article ID: 065205. https://doi.org/10.1103/PhysRevC.65.065205

[8] Cardarelli, F., Pace, E., Salme, G. and Simula, S. (1995) Physics Letters B, 357, 267. https://doi.org/10.1016/0370-2693(95)00921-7

[9] Casptick, S. and Isgur, S. (1986) Physical Review D, 34, 2809. https://doi.org/10.1103/PhysRevD.34.2809

[10] Glozman, L.Y., Papp, Z., Plessas, W., Vaega, K. and Wagenbrunn, R.F. (1998) Physical Review C, 57, 3406. https://doi.org/10.1103/PhysRevC.57.3406

[11] Glozman, L.Y., Plessas, W., Varga, K. and Wagenbrunn, R.F. (1998) Physical Review D, 58, Article ID: 094030. https://doi.org/10.1103/PhysRevD.58.094030

[12] Wagenbrunn, R.F., Boffi, S., Klink, W., Plessas, W. and Radici, M. (2001) Physics Letters B, 511, 31. https://doi.org/10.1016/S0370-2693(01)00622-0

[13] Boffi, S., Glozman, L.Y., Klink, W., Plessas, W., Radici, M. and Wagenbrunn, R.F. 
(2002) The European Physical Journal A, 14, 17.

[14] Wagenbrunn, R.F., Melde, T. and Plessas, W. (2005) Poincare-Invariant Spectator-Model Currents for Electroweak Nucleon Form Factors.

[15] Gross, F. and Agbakpe, P. (2006) Physical Review C, 73, Article ID: 015203. https://doi.org/10.1103/PhysRevC.73.015203

[16] Miller, G.A. (2002) Physical Review C, 66, 032201(R).

[17] Faessler, A., Gutsche, T., Lyubovitskij, V.E. and Pumsa-ard, K. (2006) Physical Review $D$, 73, Article ID: 114021. https://doi.org/10.1103/PhysRevD.73.114021

[18] Muller, D., Robschik, D., Geyer, B., Dittes, F.M. and Horejisi, J. (2006) Fortschritte der Physik, 42, 101. https://doi.org/10.1002/prop.2190420202

[19] Ji, X.D. (1997) Physical Review D, 55, 7114. (More thorough Treatment)

[20] Radyushkin, A.V. (1996) Physics Letters B, 380, 417. https://doi.org/10.1016/0370-2693(96)00528-X

[21] Guidal, M., Polyakov, V., Radyushkin, A.V. and Vanderhaegen, M. (2205) Physical Review D, 72, Article ID: 054013. https://doi.org/10.1103/PhysRevD.72.054013

[22] Kelly, J.J. (2002) Physical Review C, 66, Article ID: 065203. https://doi.org/10.1103/PhysRevC.66.065203

[23] Ashman, J., et al. (1988) Physics Letters B, 206, 364.

[24] Suura, H. (1978) Physical Review D, 17, 460. https://doi.org/10.1103/PhysRevD.17.469

[25] Suura, H. (1979) Physical Review D, 20, 1429. https://doi.org/10.1103/PhysRevD.20.1412

[26] Kurai, T. (2018) Results in Physics, 10, 865. https://doi.org/10.1016/j.rinp.2018.07.034

[27] Kurai, T. (2014) Progress of Theoretical and Experimental Physics, 2014, 053B01.

[28] Kurai, T. (2017) Results in Physics, 7, 2066. https://doi.org/10.1016/j.rinp.2017.05.028

[29] Mitra, A.N. and Kumari, I. (1977) Physical Review D, 15, 261. https://doi.org/10.1103/PhysRevD.15.261

[30] Galster, S., Klein, H., Moritz, J., Schmidt, D., Wegener, D. and Bleckwenn, J. (1971) Nuclear Physics B, 32, 221. https://doi.org/10.1016/0550-3213(71)90068-X

[31] Schmieden, H. (1999) Form Factors of Neutron. In: Menze, D.W. and Metsch, B., Eds., Proceeding of the 8 th International Conference on the Structure of Baryons, World Scientific, Singapore, 356.

[32] Bijker, R. and Iachello, F. (2004) Physical Review C, 69, Article ID: 068201. https://doi.org/10.1103/PhysRevC.69.068201

[33] Cloet, I.C., Eichmann, G., El-Bennich, B., Klahn, T. and Roberts, C.D. (2009) Few-Body Systems, 46, 1. https://doi.org/10.1007/s00601-009-0015-x

[34] Arrington, J. (2016) QCD Bound State Workshop Report Argonne National Lab.

[35] Thomas, A.W. (2008) Interplay of Spin and Orbital Angular Momentum in Proton. arXiv:0803.2775vl [hep-ph] 


\section{Appendix}

Here we show that Gell-mann Nishijima relation still holds under baryon number 0 case.

For mesons, Isospin $I$, component of Isospin $I_{3}$, strangeness $S$ are given as Table 1 .

Because Gell-mann Nishijima relation is $Q=I_{3}+\frac{1}{2}(B+S)$ ( $B$ is baryon number and $S$ is strangeness), this relation holds for meson case because of $B=$ 0 .

Reminding the fact that field theory shows the duality, we have to add up negative charge of proton $p^{-}, \Xi^{+}, \Xi^{*+}, \Omega^{+}$to the baryon list. Then using values of Table 1, we can define Table 2 and Table 3 for baryons.

Then it is easy to notice that Gell-mann Nishijima relation also holds for baryon case under the baryon number $B=0$. This means that baryon number 1 is not necessary.

The verification of the meson pair of each baryons shown in Table 2 and $\mathrm{Ta}$ ble 3 are given elsewhere by baryon mass spectra and decay modes.

Table 1. Mesons.

\begin{tabular}{cccccccc}
\hline particle & $\pi^{+}$ & $\pi^{0}$ & $\pi^{-}$ & $f^{0}$ & $\eta^{0}$ & $k^{+}$ & $k^{-}$ \\
\hline$I$ & 1 & 1 & 1 & 0 & 0 & $\frac{1}{2}$ & $\frac{1}{2}$ \\
$I_{3}$ & 1 & 0 & -1 & 0 & 0 & $\frac{1}{2}$ & $-\frac{1}{2}$ \\
strangeness & 0 & 0 & 0 & 0 & 0 & 1 & -1 \\
\hline
\end{tabular}

Table 2. Spin $1 / 2$ baryons.

\begin{tabular}{|c|c|c|c|c|c|}
\hline particle & antiparticle & Meson pair & $I$ & $I_{3}$ & Strangeness \\
\hline$p^{+}$ & $p^{-}$ & $\pi^{+}+\pi^{0}$ & 1 & 1 & 0 \\
\hline$n^{0}\left(p^{0}\right)$ & $n^{0}\left(p^{0}\right)$ & $\pi^{+}+\pi^{-}$ & 1 & 0 & 0 \\
\hline$p^{-}$ & $p^{+}$ & $\pi^{-}+\pi^{0}$ & 1 & -1 & 0 \\
\hline$\Lambda^{0}$ & $\Lambda^{0}$ & $\begin{array}{c}\pi^{+}+k^{-} \\
\text {or } \pi^{-}+k^{+}\end{array}$ & 0 & 0 & 0 \\
\hline$\Sigma^{+}$ & $\Sigma^{-}$ & $\pi^{+}+\eta^{0}$ & 1 & 1 & 0 \\
\hline$\Sigma^{0}$ & $\Sigma^{0}$ & $\pi^{0}+\eta^{0}$ & 1 & 0 & 0 \\
\hline$\Sigma^{-}$ & $\Sigma^{+}$ & $\pi^{-}+\eta^{0}$ & 1 & -1 & 0 \\
\hline$\Xi^{+}$ & $\Xi^{-}$ & $\pi^{0}+k^{+}$ & $\frac{1}{2}$ & $\frac{1}{2}$ & 1 \\
\hline$\Xi^{-}$ & $\Xi^{+}$ & $\pi^{0}+k^{-}$ & $\frac{1}{2}$ & $-\frac{1}{2}$ & -1 \\
\hline$\Xi^{0}$ & $\Xi^{0}$ & $\begin{array}{c}\pi^{+}+k^{-} \\
\text {or } \pi^{-}+k^{+}\end{array}$ & 0 & 0 & 0 \\
\hline
\end{tabular}

Note that we use total sum for $\Lambda^{0}$ and $\Xi^{0}$ cases. 
Table 3. Spin 3/2 baryons.

\begin{tabular}{|c|c|c|c|c|c|}
\hline particle & antiparticle & Meson pair & $I$ & $I_{3}$ & strangeness \\
\hline$\Delta^{+}$ & $\Delta^{-}$ & $\pi^{+}+f^{0}$ & 1 & 1 & 0 \\
\hline$\Delta^{0}$ & $\Delta^{0}$ & $\pi^{0}+f^{0}$ & 1 & 0 & 0 \\
\hline$\Delta^{-}$ & $\Delta^{+}$ & $\pi^{-}+f^{0}$ & 1 & -1 & 0 \\
\hline$\Sigma^{*+}$ & $\Sigma^{*-}$ & $\pi^{+}+\eta^{0}$ & 1 & 1 & 0 \\
\hline$\Sigma^{* 0}$ & $\Sigma^{* 0}$ & $\pi^{0}+\eta^{0}$ & 1 & 0 & 0 \\
\hline$\Sigma^{*-}$ & $\Sigma^{*+}$ & $\pi^{-}+\eta^{0}$ & 1 & -1 & 0 \\
\hline$\Xi^{*+}$ & $\Xi^{*-}$ & $\eta^{0}+k^{+}$ & $\frac{1}{2}$ & $\frac{1}{2}$ & 1 \\
\hline$\Xi^{*-}$ & $\Xi^{*+}$ & $\eta^{0}+k^{-}$ & $\frac{1}{2}$ & $-\frac{1}{2}$ & -1 \\
\hline$\Xi^{* 0}$ & $\Xi^{* 0}$ & $k^{+}+k^{-}$ & 0 & 0 & 0 \\
\hline$\Omega^{+}$ & $\Omega^{-}$ & $\eta^{0}+k^{+}$ & $\frac{1}{2}$ & $\frac{1}{2}$ & 1 \\
\hline$\Omega^{-}$ & $\Omega^{+}$ & $\eta^{0}+k^{-}$ & $\frac{1}{2}$ & $-\frac{1}{2}$ & -1 \\
\hline
\end{tabular}

